\title{
Influence of Sorafenib, Sunitinib and Capecitabine on the Antioxidant Status of the Skin
}

\author{
HARALD FUSS ${ }^{1}$, JUERGEN LADEMANN $^{2}$ and SORA JUNG ${ }^{2}$ \\ ${ }^{1}$ Oncology and Palliative Care, Department of Hematology, Helios Klinikum Bad Saarow, Bad Saarow, Germany; \\ ${ }^{2}$ Department of Dermatology, Venereology and Allergology, Center of Experimental and Applied Cutaneous Physiology, \\ Charité - Universitätsmedizin Berlin, Berlin, Germany
}

\begin{abstract}
Background/Aim: The aim of the present study was to examine the effect of orally administered sorafenib $\left(\right.$ Nexavar $\left.^{\circledR}\right)$, sunitinib $\left(\right.$ Sutent $\left.{ }^{\circledR}\right)$ and capecitabine $\left(\right.$ Xeloda $\left.^{\circledR}\right)$, which cause palmoplantar erythrodysesthesia (PPE), on the antioxidant status of the skin and the formation of free radicals. Patients and Methods: A total of 42 patients were enrolled, of which 36 (85\%) completed the study. Overall, 19 received capecitabine (2,000-4,000 mg per day), 10 sunitinib (25-50 $\mathrm{mg}$ per day) and 7 sorafenib (400-800 $\mathrm{mg}$ per day). Cutaneous carotenoids as markers of the antioxidant status of the skin were measured 1 day before the first oral administration $\left(T_{\text {base }}\right)$ and at day 18 of treatment $\left(T_{1}\right)$. Results: The mean antioxidant concentrations increased significantly in patients treated with sunitinib from $3.99 \pm 1.01$ to $4.68 \pm 1.32, p=0.047$ and sorafenib from $4.83 \pm 0.74$ to $5.3 \pm 0.78, p=0.007$. Treatment with capecitabine did not significantly increase the mean antioxidant concentration. Conclusion: Formation of free radicals may not be the underlying patho-mechanism of tyrosine kinase inhibitors (TKI)- and capecitabine-associated PPE.
\end{abstract}

Targeted therapies have become increasingly important for the treatment of malignant neoplasia. Multikinase inhibitors, such as sorafenib (Nexavar ${ }^{\circledR}$, Bayer AG, Leverkusen, Germany) or sunitinib (Sutent ${ }^{\circledR}$, Pfizer Inc., New York, New York, USA) are widely used in the treatment of cancer patients. Through inhibition of receptor tyrosine kinases

Correspondence to: Prof. Jürgen Lademann, Department of Dermatology, Venereology and Allergology, Center of Experimental and Applied Cutaneous Physiology, Charité - Universitätsmedizin Berlin, Corporate member of Freie Universität Berlin, HumboldtUniversität zu Berlin, and Berlin Institute of Health, Charitéplatz 1, 10117 Berlin, Germany. Tel: +49 30450518235, Fax: +49 30450518918, e-mail: juergen.lademann@ charite.de

Key Words: Cutaneous carotenoids, free radicals, cutaneous toxicity by chemotherapy, reflectance spectroscopy, hand- and foot syndrome, nutritional intervention.
(RTKs), they lead to decreased vascularization of the tumor and thus inhibit tumor growth and metastatic activity. Despite their effectiveness regarding multiple systemic cancers, a variety of dermal side effects and toxicities can occur during the treatment including alopecia, maculopapular rash, pigmentary abnormalities and palmoplantar erythrodysesthesia (PPE) (1-5).

PPE has been described as the most common dermal side effect of sorafenib affecting up to $48 \%$ of treated patients. PPE symptoms appeared in up to $50-60 \%$ of patients treated with capecitabine (Xeloda ${ }^{\circledR}$, F. Hoffmann-La Roche AG, Basel, Switzerland) and in up to $36 \%$ of patients treated with sunitinib (6-13). Other cytotoxic agents, which are commonly associated with PPE include, among others, 5-fluorouracil, vinorelbin, (pegylated liposomal (PEG)) doxorubicin, docetaxel, paclitaxel, cyclophosphamide, cisplatin, daunorubicin, etoposide, epirubicin and cytosine (13-15).

In general, skin reactions can strongly impair the quality of life of affected patients due to stigmatization, pain and limitations of activities in daily life (16).

PPE or hand-foot syndrome can cause a strong impairment of daily life activities due to its common localization in palmar and plantar sites requiring drug dose reduction or even discontinuation in severe cases $(13,17$, 18). Furthermore, it can occur in mechanically affected areas $(19,20)$ showing different grades of severity. Symptoms vary from erythema, swelling, numbness and hyperkeratosis to erosions, rhagades, blisters and ulcerations, causing different levels of pain in affected patients $(17,19)$.

Cooling and topical corticosteroids are often used as symptomatic treatment with limited therapeutic success. Several studies showed variable effectiveness regarding different topical treatment strategies including dimethyl sulfoxide (DMSO), vitamin E, pyridoxine or cyclooxygenase 2-inhibitors $(13,21,22)$.

The dermato-histopathological findings of several case reports indicate graft-versus-host disease-like mechanisms as well as toxic reactions in the skin and eccrine sweat 
Table I. Summary of the mean carotenoid concentration [a.u.] at Tbase and T1 in patients treated with capecitabine in monotherapy and in combination with gemcitabine or oxaliplatin, sorafenib and sunitinib.

\begin{tabular}{|c|c|c|c|c|c|}
\hline & Mean carotenoid concentration [a.u.] & $\mathrm{N}$ & Standard deviation & Standard error of the mean (SEM) & $p$-Value \\
\hline $\begin{array}{l}\text { Capecitabine mono }+ \\
\text { combined therapy } \mathrm{T}_{\text {base }}\end{array}$ & 4.2 & 19 & 0.8 & 0.2 & 0.791 \\
\hline $\begin{array}{l}\text { Capecitabine mono }+ \\
\text { combined therapy } \mathrm{T}_{1}\end{array}$ & 4.2 & 19 & & & \\
\hline Capecitabine monotherapy $\mathrm{T}_{\text {base }}$ & 4.1 & 14 & 0.9 & 0.2 & 0.879 \\
\hline Capecitabine monotherapy $\mathrm{T}_{1}$ & 4.1 & 14 & 1.0 & 0.3 & \\
\hline Sorafenib Tbase & 4.8 & 7 & 0.7 & 0.3 & 0.007 \\
\hline Sorafenib $\mathrm{T}_{1}$ & 5.3 & 7 & 0.8 & 0.3 & \\
\hline Sunitinib $\mathrm{T}_{\text {base }}$ & 3.9 & 10 & 1.0 & 0.3 & 0.047 \\
\hline Sunitinib $\mathrm{T}_{1}$ & 4.7 & 10 & 1.3 & 0.4 & \\
\hline
\end{tabular}

glands, described as syringo-metaplasia $(13,23)$. Different possible patho-mechanisms have been discussed. On the one hand, direct toxic effects as well as antigen-antibody and complement-mediated reactions are described $(6,13)$. Regarding multikinase-inhibitors, sunitinib, the inhibitor of vascular endothelial growth factor receptors, might decrease the vascular recovery of skin sites with high vascularization after moderate pressure, friction or even microtrauma $(3,6)$. Regarding doxorubicin and PEG doxorubicin, spectroscopic measurements indicated another patho-mechanism involving the secretion of intravenously applied doxorubicin through the sweat glands followed by intense formation of free radicals and subsequent destructive effects on the skin $(24,25)$. Following this theory, free radical formation upon treatment with anticancer drugs might indicate the effectiveness of topically applied antioxidants in the prevention and therapy of PPE $(15,26,27)$.

Further PPE-associated chemotherapeutics, namely paclitaxel, docetaxel and 5-fluorouracil have been shown to induce free radical formation in the skin shortly after chemotherapeutic infusion (28) while, to our knowledge, the detection of these substances in the skin has not yet been possible. Moreover, capecitabine was recently detected in the sweat of the skin surface after oral administration using highresolution atomic absorption spectroscopy (29). Interestingly, a preventive strategy using an antioxidant ointment with a high radical protection factor showed preventive effects in patients treated with (PEG)-doxorubicin, while it failed to show superior preventive effects compared to a ureacontaining formulation in patients under treatment with capecitabine (30). Here, orally administered capecitabine, sorafenib and sunitinib, which are known to be strongly associated with PPE, were investigated with regard to their ability to alter the antioxidant status of the skin and thus radical formation.

\section{Materials and Methods}

Study design. Measurements of the antioxidant status of the skin prior to and during chemotherapy were performed in 42 cancer patients aged from 47 to 83 years old suffering from colon- $(35.3 \%)$, renal $(33.3 \%)$, pancreatic- $(11.1 \%)$, hepatocellular - (11.1\%) cancer and other cancer diseases (acute myeloid leukemia, esophageal cancer, thyroid carcinoma, cholangiocellular carcinoma) (8.9\%).

Inclusion criteria were oral treatments with either sorafenib $\left(\right.$ Nexavar ${ }^{\circledR}$ ) or sunitinib (Sutent ${ }^{\circledR}$ ) at any dose in monotherapy, capecitabine (Xeloda ${ }^{\circledR}$ or generic drugs) at any dose either in monotherapy or in combination with oxaliplatin or gemcitabine. All patients received tyrosine kinase inhibitors (TKIs) in a palliative setting; $30 \%$ of patients treated with capecitabine received adjuvant treatment.

The base value measurements of the antioxidant status of the skin were performed on the day prior to the first oral administration at the timepoint $\mathrm{T}_{\text {base }}$ and 10 to 18 days after the first treatment day within the first cycle of chemotherapy $\left(T_{1}\right)$. The follow-up measurement was performed 10 weeks $\left(\mathrm{T}_{2}\right)$ after the first treatment day in patients treated with capecitabine either in monotherapy or in combination with oxaliplatin or gemcitabine. Side effects, such as fatigue, nausea, emesis or other toxicities were assessed at each time point.

The patients were advised not to take any antioxidants or other complementary substances. All patients received nutritional counseling at each visit. They were advised to adhere to a highenergy diet with fruits and vegetables while abstaining from consuming possible noxae.

Written informed consent was obtained from all participating patients. The study had been approved by the Ethics Committee of the Charite - Universitätsmedizin Berlin (EA1/235/14) and was performed according to the declaration of Helsinki.

Analysis of antioxidant status of the skin. Cutaneous carotenoids were measured in vivo as markers of the antioxidant status of the skin as shown previously $(31,32)$. Measurements were performed using a non-invasive miniaturized mobile system based on reflectance spectroscopy (Biozoom ${ }^{\circledR}$, Biozoom services $\mathrm{GmbH}$, Kassel, Germany). The device had been calibrated based on previous results using resonance Raman spectroscopy and has 


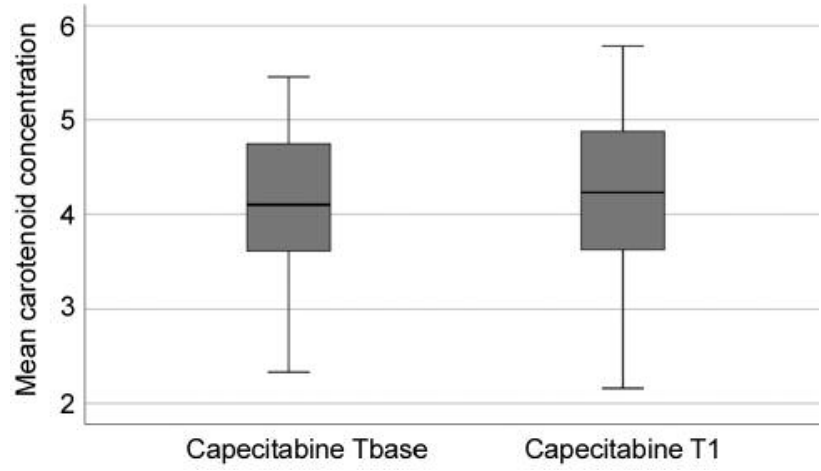

Figure 1. Comparison of mean carotenoid concentrations at $T_{\text {base }}$ and $T_{1}$ in patients treated with capecitabine in monotherapy and in combination with gemcitabine or oxaliplatin.

already been described and applied in several in vivo studies in the past (33-35). All measurements were performed on the thenar eminence of the palms of each patient performing three single measurements at each time point. Measurement results of the carotenoid concentration of the skin were displayed on an arbitrary scale from 0 to 10 and statistically analyzed conducting nonparametrical testing using SPSS v.25 (IBM corp., Armonk, NY, USA) (36).

\section{Results}

A total of 42 cancer patients (23 male and 19 female) were enrolled in the study. The dropout rate was $14 \%$ (6 of 42 ).

The mean carotenoid concentrations of the patients who completed the study for the three chemotherapeutic agents are summarized in Table I. The boxplots of the results are presented in Figures 1-3.

The mean concentration of carotenoids at $T_{\text {base }}$ prior to initiation of therapy, and at day 18 after the first day of treatment with capecitabine monotherapy were $4.11 \pm 0.88$ a.u. and $4.10 \pm 0.96$ a.u., respectively.

An increase of the mean concentration of carotenoids from $4.4 \pm 0.67$ a.u. to $4.59 \pm 0.8$ a.u. $(p=0.179)$ was found during treatment with capecitabine combined with oxaliplatin or gemcitabine. This increase showed no statistical significance.

The mean concentration of carotenoids increased significantly from $3.94 \pm 1.01$ a.u. to $4.68 \pm 1.32$ a.u. $(p=0.047)$ after sunitinib administration.

In patients treated with sorafenib the mean concentration of carotenoids increased significantly from $4.83 \pm 0.74$ a.u. to $5.3 \pm 0.78$ a.u. $(p=0.007)$.

The mean concentration of carotenoids increased insignificantly from $4.53 \pm 0.62$ a.u. at $\mathrm{T}_{\text {base prior to }}$ capecitabine treatment to $4.83 \pm 0.98$ a.u. at 10 weeks after beginning of treatment with capecitabine (Table II).

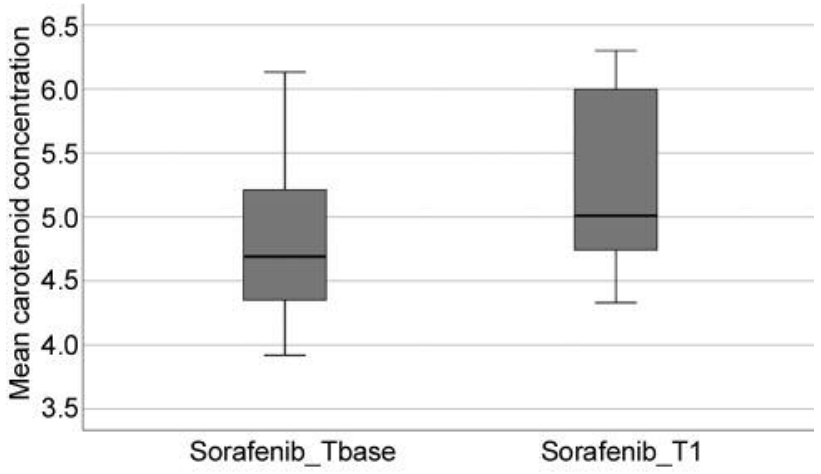

Figure 2. Comparison of mean carotenoid concentrations at $T_{\text {base }}$ and $T_{1}$ in patients treated with sorafenib.

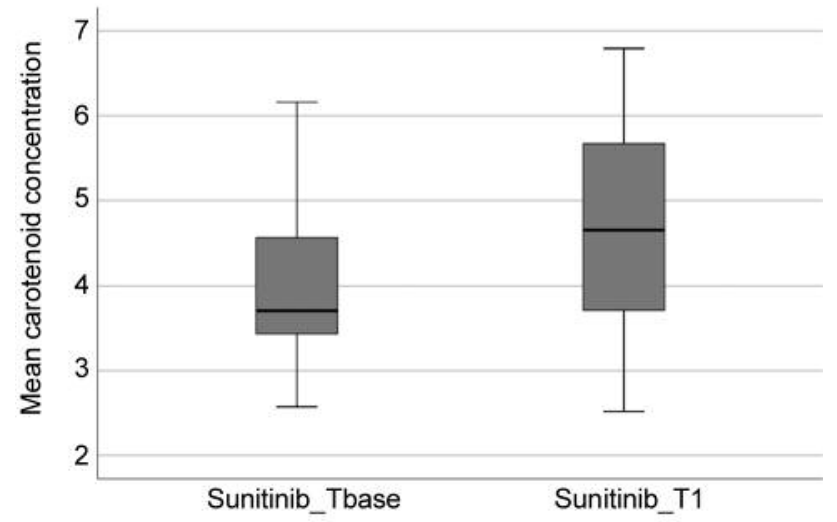

Figure 3. Comparison of mean carotenoid concentrations at $T_{\text {base }}$ and $T_{1}$ in patients treated with sunitinib.

The dropout rate was $14 \%$ (6 of 42 patients) due to adverse events between $\mathrm{T}_{\text {base }}$ and $\mathrm{T}_{1}$. Further 9 patients (25\%) discontinued the study after 9 to 12 weeks between $\mathrm{T}_{1}$ and $\mathrm{T}_{2}$. Reasons for the drop-outs were discontinuation of treatment, death or hospitalization. The decision for treatment termination was also based on clinical criteria corresponding to CTCAE > grade 2 (37).

Especially sorafenib treatment led to a high drop-out rate due to toxicity between $\mathrm{T}_{1}$ and $\mathrm{T}_{2}$ ( $85 \%$ of patients). There was no significant difference in carotenoid concentrations at $\mathrm{T}_{\text {base }}$ between the different treatment groups or with regard to the diagnosed cancerous disease, age or sex.

One patient treated with capecitabine developed PPE grade III in the 5th week after initiation of therapy, which led to termination of the treatment. The overall occurrence of PPE grade I to III is summarized in Table III. 
Table II. Mean concentration of carotenoids between $T_{\text {base }}$ and $T_{2}$ for patients treated with capecitabine in monotherapy and combination with oxaliplatin or gemcitabine.

\begin{tabular}{lccccc}
\hline & Mean carotenoid concentration & $\mathrm{N}$ & Standard deviation & Standard error of the mean (SEM) & $p$-Value \\
\hline Capecitabine $\mathrm{T}_{\text {base }}$ & 4.53829 & 7 & 0.628939 & 0.237717 \\
Capecitabine $\mathrm{T}_{2}$ & 4.82900 & 7 & 0.986636 & 0.372913 \\
\hline
\end{tabular}

Table III. PPE incidence in patients receiving capecitabine, sorafenib and sunitinib.

\begin{tabular}{lccccc}
\hline & \multicolumn{3}{c}{ PPE } & Total & Drop-out \\
\cline { 2 - 4 } & Grade I & Grade II & Grade III & & \\
\hline Capecitabine monotherapy & 0 & 2 & 0 & 3 & 0 \\
Capecitabine combination therapy & 0 & 0 & 0 & 0 & 0 \\
Sorafenib & 0 & 0 & 0 & 1 & 0 \\
Sunitinib & 0 & 1 & & & \\
\hline
\end{tabular}

\section{Discussion}

Although it has been shown that capecitabine is detectable on the skin surface 10 days after initiation of oral administration (29), here, no significant change in the antioxidant status of the skin was found within the same time period. Therefore, it can be assumed that, in contrast to doxorubicin, where topically applied antioxidants were proven to have significant preventive effects, the pathomechanism of capecitabine-induced PPE may not involve free radical formation in the skin. Since no significant decrease in the antioxidant status of the skin was measured within this study regarding all three orally administered chemotherapeutic agents, it can be assumed that the underlying patho-mechanism in capecitabine-, sunitinib- and sorafenib- associated PPE is not based on free radical formation. Previous investigations focused on changes of the antioxidant status after intravenous administration of paclitaxel, docetaxel and 5-fluorouracil, presented significant variations und (28). The effects of orally administered drugs can differ due to metabolic effects. Further investigations are needed to explore the underlying patho-mechanism of PPE due to orally and intravenously administered chemotherapy.

The relatively high drop-out rate in TKI patients is mainly a result of including patients with an ECOG status of 2-3 in the study. Especially in the sorafenib group, the treatment of patients with hepatocellular carcinoma (HCC) and advancedstage cancers of the liver and the pancreas is responsible for the increased occurrence of toxicity and the necessity of clinical treatment modification.
Despite the expansion of the measuring period until $\mathrm{T}_{2}$, a response evaluation according to RECIST criteria (which is already not standardized for targeted therapies) was not possible (38). The termination of treatment was performed in accordance with the clinical progression.

In this study the patients were closely supervised and clinically monitored and immediate intervention was applied if necessary. The consultation lasted 30-45 min per visit and patient, and included nutritional counseling and a BIA measurement in $70 \%$ of capecitabine patients and $60 \%$ of patients under TKI treatment. Studies have shown that the described interventional strategy might not influence the patient's clinical outcome but most likely has an effect on the antioxidative potential (39). Here, the changes in the nutritional behavior can be assumed to have led to the elevation of the antioxidant status of the skin during chemotherapy treatment.

A standardized evaluation of subjective life quality was not performed. Previous investigations showed an increase in antioxidants due to positive thinking and optimism (40). Clinically the increase in antioxidative potential in the sorafenib and sunitinib groups until $\mathrm{T}_{1}$ was reproducible. Particularly remarkable is the relatively low rate of PPE occurrence. We estimate that this is due to the close supervision of patient adherence to suggested behavioral measures, for example the avoidance of mechanical friction or pressure, as well as the use of topical urea-containing formulations (excluding at the time of measurement). At the same time, it has been shown that the used measurement method is easily applicable and practicable on patient-side under clinical conditions (41). 
Further analysis of immune checkpoint therapies as well as multikinase inhibitors that are not exclusive to the VEGFR pathway, which are both known to cause analogous cutaneous toxicity, is advised.

\section{Conflicts of Interest}

All Authors involved declare no conflict of interest regarding this study.

\section{References}

1 Belum VR, Washington C, Pratilas CA, Sibaud V, Boralevi F and Lacouture ME: Dermatologic adverse events in pediatric patients receiving targeted anticancer therapies: A pooled analysis. Pediatric Blood Cancer 62(5): 798-806, 2015.

2 Senapati J, Devasia AJ, Ganapule A, George L and Viswabandya A: Sorafenib induced hand foot skin rash in flt 3 itd mutated acute myeloid leukemia-a case report and review of literature. Mediterr J Hematol Infect Dis 6(1): e2014016, 2014.

3 McLellan B and Kerr H: Cutaneous toxicities of the multikinase inhibitors sorafenib and sunitinib. Dermatol Ther 24(4): 396400, 2011.

4 Autier J, Escudier B, Wechsler J, Spatz A and Robert C: Prospective study of the cutaneous adverse effects of sorafenib, a novel multikinase inhibitor. Arch Dermatol 144(7): 886-892, 2008 .

5 Lipworth AD, Robert C and Zhu AX: Hand-foot syndrome (hand-foot skin reaction, palmar-plantar erythrodysesthesia): Focus on sorafenib and sunitinib. Oncology 77(5): 257-271, 2009.

6 Suwattee P, Chow S, Berg BC and Warshaw EM: Sunitinib: A cause of bullous palmoplantar erythrodysesthesia, periungual erythema, and mucositis. Arch Dermatol 144(1): 123-125, 2008.

7 Rock EP, Goodman V, Jiang JX, Mahjoob K, Verbois SL, Morse D, Dagher R, Justice R and Pazdur R: Food and drug administration drug approval summary: Sunitinib malate for the treatment of gastrointestinal stromal tumor and advanced renal cell carcinoma. Oncologist 12(1): 107-113, 2007.

8 Garcia JA, Hutson TE, Elson P, Cowey CL, Gilligan T, Nemec C, Dreicer R, Bukowski RM and Rini BI: Sorafenib in patients with metastatic renal cell carcinoma refractory to either sunitinib or bevacizumab. Cancer 116(23): 5383-5390, 2010.

9 Lassere Y and Hoff P: Management of hand-foot syndrome in patients treated with capecitabine (xeloda). Eur J Oncol Nurs 8(Suppl 1): S31-40, 2004.

10 Gressett SM, Stanford BL and Hardwicke F: Management of hand-foot syndrome induced by capecitabine. J Oncol Pharm Pract 12(3): 131-141, 2006.

11 Rosenbaum SE, Wu S, Newman MA, West DP, Kuzel T and Lacouture ME: Dermatological reactions to the multitargeted tyrosine kinase inhibitor sunitinib. Support Care Cancer 16(6): 557-566, 2008.

12 Lee WJ, Lee JL, Chang SE, Lee MW, Kang YK, Choi JH, Moon $\mathrm{KC}$ and Koh JK: Cutaneous adverse effects in patients treated with the multitargeted kinase inhibitors sorafenib and sunitinib. Br J Dermatol 161(5): 1045-1051, 2009.

13 Degen A, Alter M, Schenck F, Satzger I, Volker B, Kapp A and Gutzmer R: The hand-foot-syndrome associated with medical tumor therapy - classification and management. J Dtsch Dermatol Ges 8(9): 652-661, 2010.

14 Baack BR and Burgdorf WH: Chemotherapy-induced acral erythema. J Am Acad Dermatol 24(3): 457-461, 1991.

15 Lademann J, Rowert-Huber HJ, Haas N, Kluschke F, Patzelt A, Zastrow L, Lange-Asschenfeldt B, Jung S, Sterry W and Sehouli $\mathrm{J}$ : Palmoplantar erythrodysesthesia-like skin symptoms in patients under various chemotherapeutics: Preventive and therapeutic options. Skin Pharmacol Physiol 27(5): 229-233, 2014.

16 Hackbarth M, Haas N, Fotopoulou C, Lichtenegger W and Sehouli J: Chemotherapy-induced dermatological toxicity: Frequencies and impact on quality of life in women's cancers. Results of a prospective study. Support Care Cancer 16(3): 267273, 2008.

17 Lorusso D, Di Stefano A, Carone V, Fagotti A, Pisconti S and Scambia G: Pegylated liposomal doxorubicin-related palmarplantar erythrodysesthesia ('hand-foot' syndrome). Ann Oncol 18(7): 1159-1164, 2007.

18 Nagore E, Insa A and Sanmartin O: Antineoplastic therapyinduced palmar plantar erythrodysesthesia ('hand-foot') syndrome. Incidence, recognition and management. Am J Clin Dermatol 1(4): 225-234, 2000.

19 Jung S, Sehouli J, Patzelt A and Lademann J: Influence of mechanical stress on palmoplantar erythrodysesthesia - a case report. Oncol Res Treat 38(1-2): 42-44, 2015.

20 Sibaud V, Delord JP and Chevreau C: Sorafenib-induced handfoot skin reaction: A koebner phenomenon? Target Oncol 4(4): 307-310, 2009.

21 Anderson R, Jatoi A, Robert C, Wood LS, Keating KN and Lacouture ME: Search for evidence-based approaches for the prevention and palliation of hand-foot skin reaction (hfsr) caused by the multikinase inhibitors (mkis). Oncologist 14(3): 291-302, 2009.

22 Lacouture ME, Wu S, Robert C, Atkins MB, Kong HH, Guitart J, Garbe C, Hauschild A, Puzanov I, Alexandrescu DT, Anderson RT, Wood L and Dutcher JP: Evolving strategies for the management of hand-foot skin reaction associated with the multitargeted kinase inhibitors sorafenib and sunitinib. Oncologist 13(9): 1001-1011, 2008.

23 Valks R, Fraga J, Porras-Luque J, Figuera A, Garcia-Diez A and Fernandez-Herrera J: Chemotherapy-induced eccrine squamous syringometaplasia. A distinctive eruption in patients receiving hematopoietic progenitor cells. Arch Dermatol 133(7): 873-878, 1997.

24 Jacobi U, Waibler E, Schulze P, Sehouli J, Oskay-Ozcelik G, Schmook T, Sterry W and Lademann J: Release of doxorubicin in sweat: First step to induce the palmar-plantar erythrodysesthesia syndrome? Ann Oncol 16(7): 1210-1211, 2005.

25 Martschick A, Sehouli J, Patzelt A, Richter H, Jacobi U, OskayOzcelik G, Sterry W and Lademann J: The pathogenetic mechanism of anthracycline-induced palmar-plantar erythrodysesthesia. Anticancer Res 29(6): 2307-2313, 2009.

26 Kluschke F, Martschick A, Darvin ME, Zastrow L, Chekerov R, Lademann J and Sehouli J: Application of an ointment with high radical protection factor as a prevention strategy against ppe. $\mathrm{J}$ Clin Oncol 30(15), 2012.

27 Lademann J, Martschick A, Kluschke F, Richter H, Fluhr JW, Patzelt A, Jung S, Chekerov R, Darvin ME, Haas N, Sterry W, Zastrow L and Sehouli J: Efficient prevention strategy against the development of a palmar-plantar erythrodysesthesia during chemotherapy. Skin Pharmacol Physiol 27(2): 66-70, 2014. 
28 Lee BN, Jung S, Darvin ME, Eucker J, Kuhnhardt D, Sehouli J, Chekerov R, Patzelt A, Fuss H, Yu RX and Lademann J: Influence of chemotherapy on the antioxidant status of human skin. Anticancer Res 36(8): 4089-4093, 2016.

29 Huang MD, Fuss H, Lademann J, Florek S, Patzelt A, Meinke MC and Jung S: Detection of capecitabine (xeloda(r)) on the skin surface after oral administration. J Biomed Opt 21(4): 47002, 2016.

30 Hofheinz RD, Gencer D, Schulz H, Stahl M, Hegewisch-Becker S, Loeffler LM, Kronawitter U, Bolz G, Potenberg J, Tauchert F, Al-Batran SE and Schneeweiss A: Mapisal versus urea cream as prophylaxis for capecitabine-associated hand-foot syndrome: A randomized phase iii trial of the aio quality of life working group. J Clin Oncol 33(22): 2444-U2452, 2015.

31 Darvin ME, Sandhagen C, Koecher W, Sterry W, Lademann J and Meinke MC: Comparison of two methods for noninvasive determination of carotenoids in human and animal skin: Raman spectroscopy versus reflection spectroscopy. J Biophotonics 5(7): 550-558, 2012.

32 Lademann J, Schanzer S, Meinke M, Sterry W and Darvin ME: Interaction between carotenoids and free radicals in human skin. Skin Pharmacol Physiol 24(5): 238-244, 2011.

33 Darvin ME, Patzelt A, Knorr F, Blume-Peytavi U, Sterry W and Lademann J: One-year study on the variation of carotenoid antioxidant substances in living human skin: Influence of dietary supplementation and stress factors. J Biomed Opt 13(4): 044028, 2008.

34 Jung S, Darvin ME, Chung HS, Jung B, Lee SH, Lenz K, Chung WS, Yu RX, Patzelt A, Lee BN, Sterry W and Lademann J: Antioxidants in asian-korean and caucasian skin: The influence of nutrition and stress. Skin Pharmacol Physiol 27(6): 293-302, 2014.

35 Lademann J, Köcher W, Yu R, Meinke MC, Na Lee B, Jung S, Sterry W and Darvin ME: Cutaneous carotenoids: The mirror of lifestyle? Skin Pharmacol Physiol 27(4): 201, 2014.
$36 \mathrm{Ibm}$ analytics spss trials [internet]. Last updated 06/2017 [quoted 01/2018]. IBM URL: https://wwwibmcom/analytics/de/de/ technology/spss/spss-trialshtml,

37 National Institutes of Health: National Cancer Institute. Common Terminology Criteria for Adverse Events (CTCAE) (v4.03). NIH Publication No.09-5410,

38 Eisenhauer EA, Therasse P, Bogaerts J, Schwartz LH, Sargent D, Ford R, Dancey J, Arbuck S, Gwyther S, Mooney M, Rubinstein L, Shankar L, Dodd L, Kaplan R, Lacombe D and Verweij J: New response evaluation criteria in solid tumours: Revised recist guideline (version 1.1). Eur J Cancer 45(2): 228247, 2009.

39 Bourdel-Marchasson I, Blanc-Bisson C, Doussau A, Germain C, Blanc JF, Dauba J, Lahmar C, Terrebonne E, Lecaille C, Ceccaldi J, Cany L, Lavau-Denes S, Houede N, Chomy F, Durrieu J, Soubeyran P, Senesse P, Chene G and Fonck M: Nutritional advice in older patients at risk of malnutrition during treatment for chemotherapy: A two-year randomized controlled trial. Plos One 9(9), 2014.

40 Boehm JK, Williams DR, Rimm EB, Ryff C and Kubzansky LD: Association between optimism and serum antioxidants in the mid life in the united states study. Psychosomatic Med 75(1): 2$10,2013$.

41 Xing YL, Fuss H, Lademann J, Huang MD, Becker-Ross H, Florek S, Patzelt A, Meinke MC, Jung S and Esser N: A new concept of efficient therapeutic drug monitoring using the highresolution continuum source absorption spectrometry and surface enhanced raman spectroscopy. Spectrochim Acta Part B At Spectrosc 142: 91-96, 2018.
Received July 31, 2018

Revised August 16, 2018 Accepted August 19, 2018 